\title{
Long-chain fatty acid activates hepatocytes through CD36 mediated oxidative stress
}

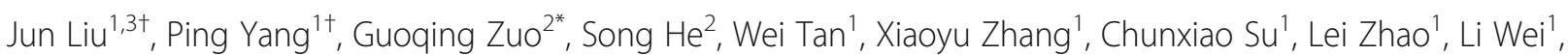 \\ Yao Chen ${ }^{1}$, Xiongzhong Ruan ${ }^{1,4,5}$ and Yaxi Chen ${ }^{*^{*}}$
}

\begin{abstract}
Background: Accumulating evidence suggests that activated hepatocytes are involved in the deposition of the excess extracellular matrix during liver fibrosis via the epithelial to mesenchymal transition. Lipid accumulation in hepatocytes are implicated in the pathogenesis of chronic liver injury. CD36 is known to mediate long-chain fatty acid (LCFA) uptake and lipid metabolism. However, it is unclear whether LCFA directly promotes hepatocyte activation and the involved mechanisms have not been fully clarified.
\end{abstract}

Methods: Mice were fed with a high fat diet (HFD) and normal hepatocyte cells (Chang liver cells) were treated with palmitic acid (PA) in vivo and in vitro. Real-time polymerase chain reaction (RT-PCR) and western blotting were used to examine the gene and protein expression of molecules involved in hepatic fibrogenesis and hepatocyte activation. CD36 was knocked down by transfecting CD36 siRNA into hepatocyte cells. Hydrogen peroxide $\left(\mathrm{H}_{2} \mathrm{O}_{2}\right)$ and reactive oxygen species (ROS) levels were detected using commercial kits.

Results: HFD induced a profibrogenic response and up-regulated CD36 expression in vivo. Analogously, PA increased lipid accumulation and induced human hepatocyte activation in vitro, which was also accompanied by increased CD36 expression. Interestingly, knockdown of CD36 resulted in a reduction of hepatocyte lipid deposition and decreased expression of Acta2 (34\% decrease), Vimentin (29\% decrease), Desmin (60\% decrease), and TGF- $\beta$ signaling pathway related genes. In addition, HFD and PA increased the production of $\mathrm{H}_{2} \mathrm{O}_{2}$ in vivo (48\% increase) and in vitro (385\% increase), and the antioxidant, NAC, ameliorated PA-induced hepatocyte activation. Furthermore, silencing of CD36 in vitro markedly attenuated PA-induced oxidative stress $\left(\mathrm{H}_{2} \mathrm{O}_{2}: 41 \%\right.$ decrease; ROS: $39 \%$ decrease), and the anti-activation effects of CD36 knockdown could be abolished by pretreatment with $\mathrm{H}_{2} \mathrm{O}_{2}$.

Conclusions: Our study demonstrated that LCFA facilitates hepatocyte activation by up-regulating oxidative stress through CD36, which could be an important mechanism in the development of hepatic fibrosis.

Keywords: CD36, LCFA, Hepatocyte activation, Oxidative stress

\section{Background}

Liver cirrhosis is the end-stage condition of various chronic liver diseases, and fibrosis is the precursor of cirrhosis, which is also considered a severe disease $[1,2]$. Liver fibrosis is a reversible wound healing response to liver injury and is considered to be a pathological process

\footnotetext{
*Correspondence: cqzgqly@163.com; chenyaxi@cqmu.edu.cn

† Jun Liu and Ping Yang contributed equally to this work.

${ }^{2}$ Department of Gastroenterology, Chongqing Hospital of Traditional Chinese Medicine, Chongqing 400021, China

${ }^{1}$ Centre for Lipid Research \& Key Laboratory of Molecular Biology for Infectious Diseases (Ministry of Education), Institute for Viral Hepatitis, Department of Infectious Diseases, The Second Affiliated Hospital, Chongqing Medical University, Chongqing 400016, China Full list of author information is available at the end of the article
}

characterized by the production and excessive deposition of extracellular matrix (ECM) [3, 4]. Liver fibrogenic cells participate in the process via different mechanisms of the epithelial-mesenchymal transition (EMT) when epithelial cells lose their original characteristics and gradually obtain a mesenchymal phenotype, and this transition can be called activation [5, 6]. Even though activated hepatic stellate cells (HSCs) are the most important source of ECM proteins during the fibrogenesis [7], a wealth of evidence has confirmed that hepatocytes are involved in EMT during hepatic fibrosis, and they have been considered to be another type of myofibroblast-like cells [8]. It is

(c) The Author(s). 2018 Open Access This article is distributed under the terms of the Creative Commons Attribution 4.0 International License (http://creativecommons.org/licenses/by/4.0/), which permits unrestricted use, distribution, and 
known that activated hepatocytes up-regulate the expression of typical mesenchymal cell markers, such as a-SMA, type 1 collagen, vimentin, desmin, and fibronectin, and down-regulate E-cadherin and cytokeratin $[9,10]$. TGF- $\beta$ is recognized as a potent inducer of EMT and a major cytokine in liver fibrosis that induces the profibrogenic pathway and fibrosis in the liver [11].

Excess lipid accumulation in the liver may be an important cause of pathogenesis in chronic liver injury [12]. Studies have found a high correlation between steatosis and the severity of hepatic fibrosis. The accumulation of abnormally high amounts of lipids in hepatocytes may induce increased susceptibility to secondary injury, leading to an acceleration in the process of chronic liver injury [13]. Increased free cholesterol accumulation in HSCs plays a crucial role in the progression of liver fibrosis by promoting HSC activation, which results in further accumulation of free cholesterol and exaggerates liver fibrosis in a vicious cycle [14]. Furthermore, owing to the emerging role of long-chain fatty acids (LCFA) in fibrosis, Lars et al. proved that LCFA (oleate:palmitic acid (PA) 2:1) had profibrogenic effects on LX-2 via upregulation of a-SMA and TGF- $\beta$ mRNA expression [15]. However, the role of LCFA in the regulation of hepatocyte activation is unknown.

CD36, which belongs to the class B scavenger receptors, is a transmembrane glycoprotein that serves as a facilitator of lipid transport and binds various lipids, e.g., LCFA and oxidized low-density lipoprotein (ox-LDL) [16]. Previous studies demonstrated that CD36 is involved in various diseases, such as insulin resistance, atherosclerosis, and non-alcoholic fatty liver disease (NAFLD) [17]. Additionally, our recent studies found that CD36 plays an important role in balancing the hepatitis B virus life cycle and hepatic inflammation, which could lead to a new potential therapeutic strategy for the prevention of chronic liver injury [18]. More importantly, Wilhelm et al. demonstrated that ox-LDL stimulated ECM synthesis in cultured HSCs through CD36 [19], suggesting that CD36 plays an important role in the pathogenesis of hepatic fibrogenesis.

The purpose of this study was to characterize and examine the effects of PA, a LCFA, on hepatocyte activation and explore whether CD36 was involved in the potential mechanism. Firstly, we found that PA treatment significantly increased CD36 expression and induced hepatocyte activation. Secondly, PA induced hepatocyte activation depended on the oxidative stress pathway. Thirdly, knockdown of CD36 reduced oxidative stress as well as hepatocyte activation. Based on these data, we suggested that CD36 activation by PA induces hepatocyte activation through a oxidative stress dependent pathway.

\section{Methods}

\section{Cell culture}

The Chang liver cell line was obtained from $\mathrm{BeNa} \mathrm{Cul}$ ture Collection and cultured with RPMI-1640 medium containing $10 \%$ fetal bovine serum, 100 units $/ \mathrm{ml}$ penicillin, and $100 \mu \mathrm{g} / \mathrm{ml}$ streptomycin. All experiments were carried out in serum-free RPMI-1640 medium containing $0.2 \%$ bovine serum albumin (BSA), 100 units $/ \mathrm{ml}$ penicillin, and $100 \mu \mathrm{g} / \mathrm{ml}$ streptomycin. The cells were pre-incubated in serum-free medium for $12 \mathrm{~h}$ and then subjected to PA for another $48 \mathrm{~h}$. Antioxidants $\mathrm{N}$-acetylcysteine (NAC) and hydrogen peroxide $\left(\mathrm{H}_{2} \mathrm{O}_{2}\right)$ were obtained from Beyotine Biotechnology (Beijing, China), and PA was obtained from Sigma (Poole, Dorset, UK).

\section{Animal model}

Animal care and experimental procedures were approved by the Animal Care Committees at Chongqing Medical University (Number: 2014056). Six- to eight-week-old C57BL/6 J mice were randomly assigned to receive a normal chow diet (NCD, Research Diets, $\mathrm{D} 12450 \mathrm{~B}, 10 \mathrm{kcal} \%$ from Fat, $n=5$ ) or a high-fat diet (HFD, Research Diets, D12492, $60 \mathrm{kcal} \%$ from fat, $\mathrm{n}=5$ ). Finally, the mice were killed after 14 weeks. Blood samples were taken for lipid profiles, and liver samples were collected for further assessments.

\section{Serum analysis}

Serum triglyceride (TG) concentrations were determined enzymatically with commercial kits (Jiancheng, Nanjing, China). Free fatty acid (FFA) concentrations were determined calorimetrically using commercial kits (Applygen Technologies, Beijing, China).

\section{Cell proliferation assay}

Cellular proliferation was assayed using a Cell Counting Kit-8 (CCK-8) purchased from Beyotime Biotechnology (Beijing, China). Briefly, hepatocytes were seeded at a density of $2 \times 10^{4}$ in a 96-well culture plate and treated with or without PA for $48 \mathrm{~h}$. Then, absorbance was measured at $450 \mathrm{~nm}$ via a Microplate Reader (Bio-Tek, Vermont, USA).

\section{Western blotting}

The total protein content from hepatocytes or liver was lysed using RIPA containing protease inhibitor, and the protein content was measured and normalized using a BCA Protein Assay Kit. Total proteins $(20-30 \mu \mathrm{g})$ were separated by SDS-PAGE and transferred onto PVDF membranes. After blocking with $3 \% \mathrm{BSA}$, the membranes were incubated with primary antibodies anti-CD36, 1:2000 (Novus, Colorado, USA); anti-a-SMA 1:500 (Sigma, Poole, Dorset, UK); anti-vimentin 
1:1000 (CST, Danvers, USA); and anti- $\beta$-actin, 1:3000 (ProteinTech, Wuhan, China) at $4{ }^{\circ} \mathrm{C}$ overnight and subsequently incubated with their corresponding horseradish peroxidase-labeled secondary antibodies. Finally, the blot was detected using an ECL advance Western Blotting Detection Kit (Millipore, Temecula, CA, USA). The protein relative intensity was analyzed using ImageJ software (National Institutes of Health, USA).

Real-time reverse transcription polymerase chain reaction Total RNA was homogenized from cultured cells and livers of C57BL/6 J mice using RNAiso Plus reagent (Takara, Dalian, China). cDNA synthesis and quantitative real-time PCR were performed with commercial kits (Takara, Dalian, China) using the Bio-Rad CFX Connect TM Real-Time System (Bio-Rad, Hercules, CA, USA) according to the manufacturer's instructions. $\beta$-actin served as the reference housekeeping gene in vivo and in vitro. All the primer pairs used in this study are listed in Table 1.

ROS, $\mathrm{H}_{2} \mathrm{O}_{2}$, and malondialdehyde (MDA) assays Hepatocyte intracellular ROS and $\mathrm{H}_{2} \mathrm{O}_{2}$ content was measured using the ROS and $\mathrm{H}_{2} \mathrm{O}_{2}$ Assay Kit (Beyotime, Beijing, China) according to the manufacturer's instructions and was normalized by protein concentration.

Table 1 Primers for quantitative real-time PCR

\begin{tabular}{|c|c|}
\hline Gene & Primer sequences \\
\hline Human CD36 & $\begin{array}{l}\text { Forward: 5'-CTTTGGCTTAATGAGACTGGGAC-3' } \\
\text { Reverse: 5'- GCAACAAACATCACCACACCA-3' }\end{array}$ \\
\hline Human $\beta$-actin & $\begin{array}{l}\text { Forward: 5'-GTTGTCGACGACGAGCG-3' } \\
\text { Reverse : 5'-GCACAGAGCCTCGCCTT-3' }\end{array}$ \\
\hline Human Acta2 & $\begin{array}{l}\text { Forward: 5'-CATCATGCGTCTGGATCTGG-3' } \\
\text { Reverse : 5'-GGACAATCTCACGCTCAGCA-3' }\end{array}$ \\
\hline Human Vimentin & $\begin{array}{l}\text { Forward: 5'-ACCCGCACCAACGAGAAGGT-3' } \\
\text { Reverse : 5'-ATTCTGCTGCTCCAGGAAGCG-3' }\end{array}$ \\
\hline Human TGF- $\beta$ & $\begin{array}{l}\text { Forward: 5'-AAGTTGGCATGGTAGCCCTT-3' } \\
\text { Reverse: 5'-CCCTGGACACCAACTATTGC-3' }\end{array}$ \\
\hline Human Snail2 & $\begin{array}{l}\text { Forward: 5'-GCAGTGAGGGCAAGAAAAAG-3' } \\
\text { Reverse : 5'-TCGGACCCACACATTACCTT-3' }\end{array}$ \\
\hline Human Twist1 & $\begin{array}{l}\text { Forward: 5'-TCCATTITCTCCTTCTCTGGAA-3' } \\
\text { Reverse: 5'-CCTTCTCGGTCTGGAGGAT-3' }\end{array}$ \\
\hline Human Zeb1 & $\begin{array}{l}\text { Forward: 5'-CAGTCAGCTGCATCTGTAACAC-3' } \\
\text { Reverse: 5'-CCAGGTGTAAGCGCAGAAAG-3' }\end{array}$ \\
\hline Mouse CD36 & $\begin{array}{l}\text { Forward: 5'-GAGCCATCTTTGAGCCTTCA-3' } \\
\text { Reverse: 5'-TCAGATCCGAACACAGCGTA-3' }\end{array}$ \\
\hline Mouse $\beta$-actin & $\begin{array}{l}\text { Forward: 5'-CGATGCCCTGAGGCTCTIT-3' } \\
\text { Reverse: 5'-TGGATGCCACAGGATTCCAT-3' }\end{array}$ \\
\hline Mouse Acta2 & $\begin{array}{l}\text { Forward:5'-CCAGAGCAAGAGAGGGATCCT-3' } \\
\text { Reverse: 5'-TGTCGTCCCAGTTGGTGA-3' }\end{array}$ \\
\hline Mouse Col1 & $\begin{array}{l}\text { Forward: 5'-CAACCTGGACGCCATCAAG-3' } \\
\text { Reverse: 5'-CAGACGGCTGAGTAGGGAACA-3' }\end{array}$ \\
\hline Mouse Col4 & $\begin{array}{l}\text { Forward:5'-CCGAGCCAGTCCATTTATAGAATG-3' } \\
\text { Reverse: 5'-CAGCGAAGCCAGCCAGAA-3' }\end{array}$ \\
\hline
\end{tabular}

Hepatic MDA content was evaluated using a commercial kit (Jiancheng, Nanjing, China) and was normalized by total liver protein.

\section{BODIPY 493/503 staining}

BODIPY 493/503 (4, 4- difluoro-1, 3, 5, 7- tetramethyl- 4bora- 3a, 4a- diaza- s- indacene) is a fluorescent lipophilic stain widely used to label lipid droplets in plants. Briefly, solubilized BODIPY 493/503 in DMSO at $2.5 \mathrm{mg} / \mathrm{ml}$ was stored at $-20{ }^{\circ} \mathrm{C}$ in the dark. Fixed cells were incubated with a working concentration of $0.2 \mu \mathrm{g} / \mathrm{ml}$ BODIPY staining solution in the dark for $30 \mathrm{~min}$ at $37{ }^{\circ} \mathrm{C}$. The cells were washed twice with PBS, and the stained cells were visualized using a fluorescence microscope (Zeiss, Jena, Germany).

\section{Statistical analysis}

The data are expressed as the mean \pm SEM. Statistical analysis was performed using Student's t-test when only two value sets were compared and one-way analysis of variance followed by Turkey's multiple comparison test when the data involved three or more groups. A difference was considered significant if the $P$ was less than 0.05 .

\section{Results}

HFD enhances profibrogenic gene expression, along with increased CD36 expression

Serum FFA and TG levels were increased in HFD-fed mice compared with NCD-fed mice (Fig. 1a). Hepatic mRNA expression of markers of fibrogenesis, including Acta2, Col 1, and $\mathrm{Col} 4$, was significantly up-regulated in HFD-fed mice (Fig. 1b). Additionally, we also found that HFD increased hepatic CD36 protein and mRNA expression (Fig. 1c, d).

\section{PA promotes hepatocyte activation and up-regulates CD36 expression}

Hepatocytes were treated with PA at different concentrations and times to determine the effect of PA on hepatocyte lipotoxicity and proliferation. We noticed that there was significant cytotoxicity at the $0.4 \mathrm{mmol} / \mathrm{L}$ concentration, but PA was comparatively non-toxic at the $0.1 \mathrm{mmol} / \mathrm{L}$ and $0.2 \mathrm{mmol} / \mathrm{L}$ concentrations (data not shown). Furthermore, there was no stimulatory effect of PA on hepatocyte proliferation (Fig. 2a). Following BODIPY staining, we explored the concentrations of PA that enhanced lipid accumulation in hepatocytes (Fig. 2b). We found that CD36 protein expression was increased in PA-treated hepatocytes at both $0.1 \mathrm{mmol} / \mathrm{L}$ and $0.2 \mathrm{mmol} / \mathrm{L}$ concentrations (Fig. 2c). Homoplastically, PA up-regulated the protein expression of a-SMA and VIMENTIN and the mRNA expression of Acta2, Vimentin and Desmin (Fig. 2d), which allowed hepatocytes to 

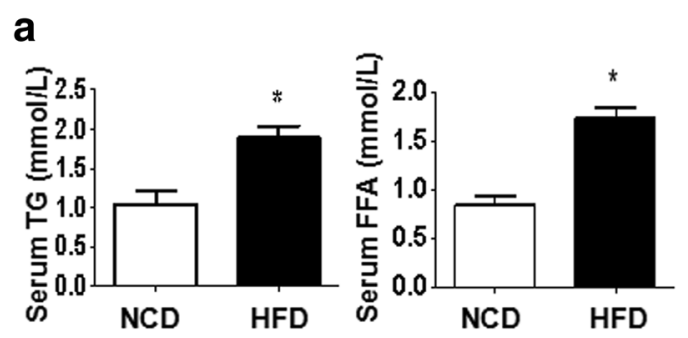

C

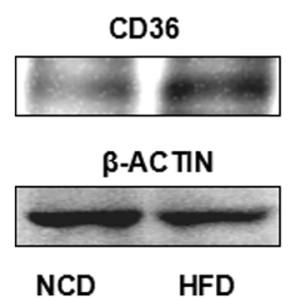

$43 K D$

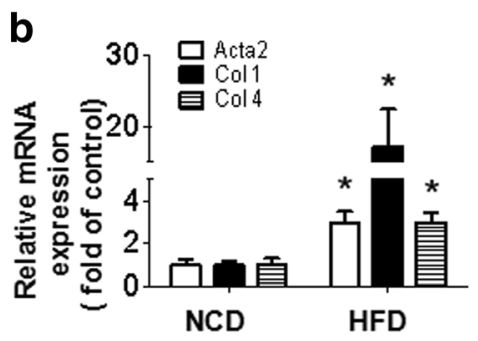

d

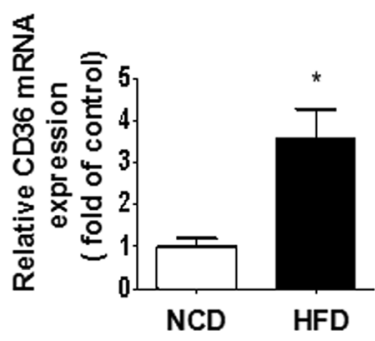

Fig. 1 Effects of HFD on profibrogenic gene and CD36 expression in the livers of C57BL/6 J mice. Mice were fed a normal chow diet (NCD) or a high-fat diet (HFD) for 14 weeks. The levels of FFA and TG in serum were measured as described in the Materials and methods (a). The hepatic mRNA expression of Acta2, Col 1, and Col 4 was determined by real-time PCR (b). The hepatic protein expression of CD36 was examined by western blotting (c). The hepatic mRNA expression of CD36 was determined by real-time PCR (d). The results are depicted as the mean \pm SEM, ${ }^{*} P<0.05$ versus the NCD group

acquire an activated phenotype. Moreover, this trend was consistent with the mRNA expression of TGF- $\beta$ and key downstream transcription factors Snail, Twist and Zeb1 (Fig. 2e). These results suggest that PA mediates the up-regulation of CD36 expression and promotes hepatocyte activation.

\section{CD36 is involved in PA-induced hepatocyte activation}

A PA concentration of $0.2 \mathrm{mmol} / \mathrm{L}$ has a marked effect on hepatocyte activation; therefore, we selected this concentration for additional experiments. We knocked down CD36 by transfecting CD36 siRNA into hepatocytes to confirm the role of CD36 in PA-induced hepatocyte activation. Our data demonstrated that CD36 siRNA transfection resulted in markedly decreased CD36 protein and mRNA expression in PA-induced hepatocytes by western blotting and real-time PCR (Fig. 3a, b), which suggested that the CD36 knockdown hepatocyte cell model has been established successfully. BODIPY staining showed that lipid accumulation clearly decreased in the CD36 knockdown group (Fig. 3c), suggesting that PA promotes hepatocyte lipid accumulation by CD36. Protein expression of activated hepatocyte markers a-SMA and VIMENTIN decreased in the CD36 knockdown group compared with the control group (Fig. 3d). Meanwhile, the mRNA expression of Acta2, Vimentin, Desmin, and TGF- $\beta$ signaling pathway related genes, such as TGF- $\beta$, Snail, and Zeb1, were also down-regulated in the CD36 knockdown group (Fig. 3e, f). These findings suggest that PA promoted hepatocyte activation was mediated by CD36.

\section{CD36 mediates PA-induced hepatocyte activation via oxidative stress}

Oxidative stress has been shown to be involved in hepatotoxicity when pro-oxidative capacity overwhelms antioxidant capacity. As shown by our results, HFD-fed mice had greater $\mathrm{H}_{2} \mathrm{O}_{2}$ and MDA production in the liver than control mice (Fig. 4a, b). Similarly, the production of $\mathrm{H}_{2} \mathrm{O}_{2}$ and ROS was considerably higher in hepatocytes treated with PA than in the control group (Fig. 4c). Knockdown of CD36 considerably decreased the production of $\mathrm{H}_{2} \mathrm{O}_{2}$ and $\mathrm{ROS}$ in hepatocytes treated with PA (Fig. 4d). These results suggested that the level of oxidative stress depends on hepatocyte CD36 expression when treated with PA. We individually applied the antioxidant NAC in PA-treated hepatocytes and $\mathrm{H}_{2} \mathrm{O}_{2}$ in PA-treated hepatocytes with CD36 knockdown to determine whether the oxidative stress mediated by CD36 was involved in PA-induced hepatocyte activation. We further found that NAC significantly decreased PA-induced hepatocyte activation and TGF- $\beta$ signaling pathway related genes (Fig. 4e, f). In addition, the $\mathrm{H}_{2} \mathrm{O}_{2}$ supplement largely abrogated the improved effect of CD36 knockdown on hepatocyte activation (Fig. 4g-i). These data suggest that oxidative stress is critical in PA-induced hepatocyte activation and that this activation is mediated by CD36.

\section{Discussion}

The purpose of our current study was to clarify the effects of lipids on hepatocyte activation. Here, we show 

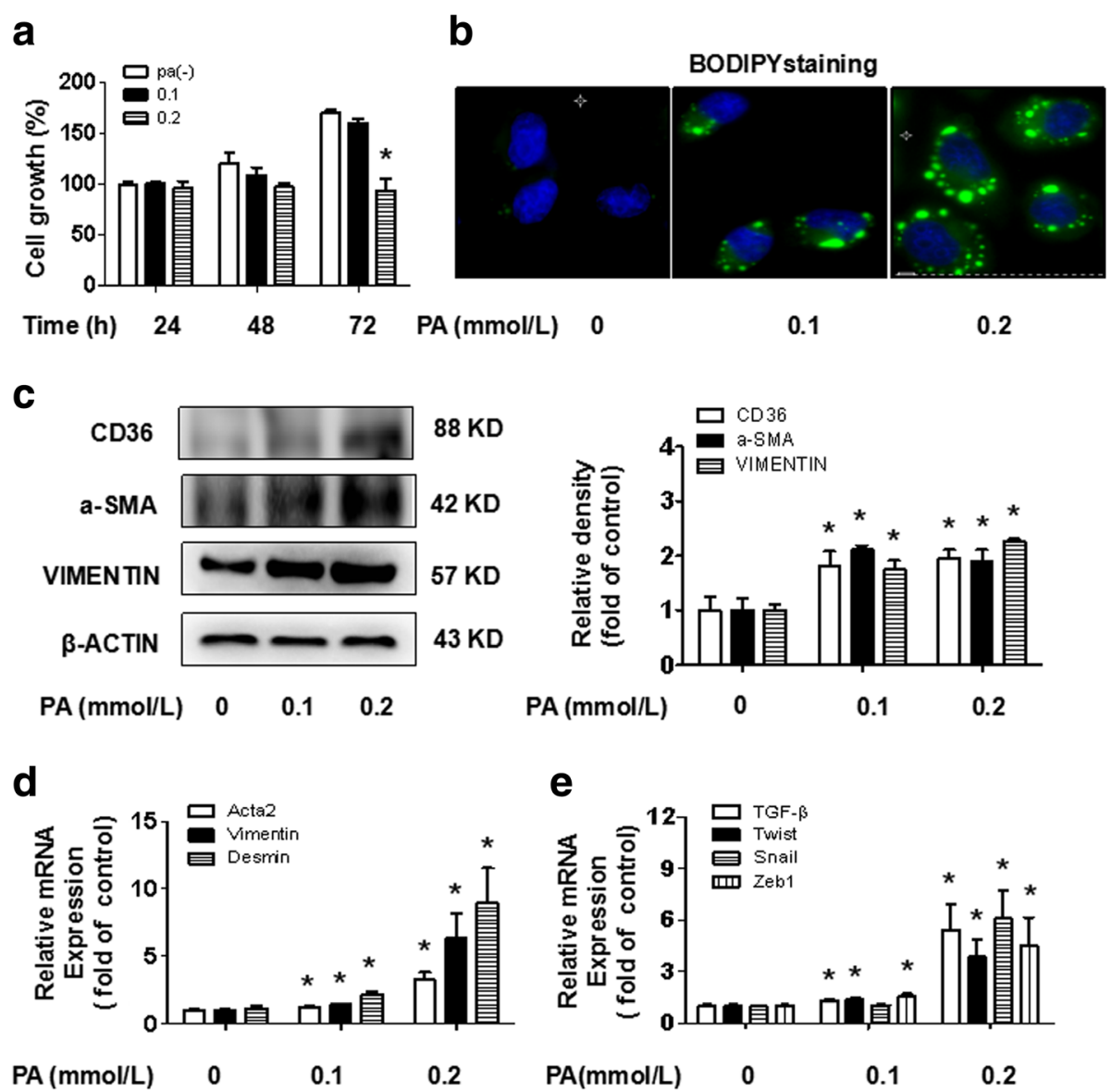

Fig. 2 Effects of PA on hepatocyte activation and CD36 expression. Hepatocytes were incubated in serum-free medium containing different concentrations of PA. After the time indicated, a CCK-8 cell proliferation assay was performed to detect hepatocyte proliferation in response to PA (a). Lipid accumulation was observed by BODIPY staining (original magnification $\times 800$, b). The protein expression of CD36, a-SMA, and VIMENTIN was examined by western blotting (c). The mRNA expression of Acta2, Vimentin, Desmin and TGF- $\beta$ signaling pathway related genes TGF- $\beta$, Snail, Twist, and Zeb1 was determined by real-time PCR $(\mathbf{d}, \mathbf{e})$. The results are depicted as the mean \pm SEM, ${ }^{*} P<0.05$ versus the 0 mmol/L PA group

that: 1) LCFA treatment induced hepatocyte activation, evidenced by up-regulated expression of Acta2, Vimentin, Desmin, and TGF- $\beta$ signaling pathway; 2) CD36 inhibition attenuated LCFA-induced hepatocyte activation; 3) Oxidative stress was critical in LCFA-induced hepatocyte activation, which was mediated by CD36.

Many studies have demonstrated the potential role of functional food ingredients in controlling dyslipidaemia and lipid metabolism both in animal models and in humans [20]. Excess lipids intake induces hyperlipidaemia and lipid accumulation in the liver that results in the development of NAFLD [21]. Previous studies have suggested a close association between lipids and liver fibrosis. Increased cholesterol intake accelerated liver fibrosis, which was mainly due to increased free cholesterol accumulation in HSCs, thus promoting HSC activation [14]. Previous studies also indicated that LCFA and ox-LDL had profibrogenic effects on HSCs via the up-regulation of ECM synthesis [15, 19]. Numerous studies have demonstrated that hepatocytes are important cells when they evolve into an activated phenotype in the development of hepatic fibrosis over decades [11, 22]. Although there are reports that FFA has a certain effect on HSC activation [15], there is a relatively poor understanding of the FFA involved in hepatocyte activation. In this study, we demonstrated that PA induces hepatocyte activation, as evidenced by increased mRNA abundance of Acta2, Vimentin and Desmin, which are representative markers of hepatocyte activation.

The activation of TGF- $\beta$ signaling pathways has been verified, and this activation could promote the subsequent activation of EMT transcription factors, including SNAIL, ZEB and TWIST, which allows epithelial cells to acquire a mesenchymal phenotype [23]. In particular, it is now accepted that TGF- $\beta$ is a crucial fibrogenic 

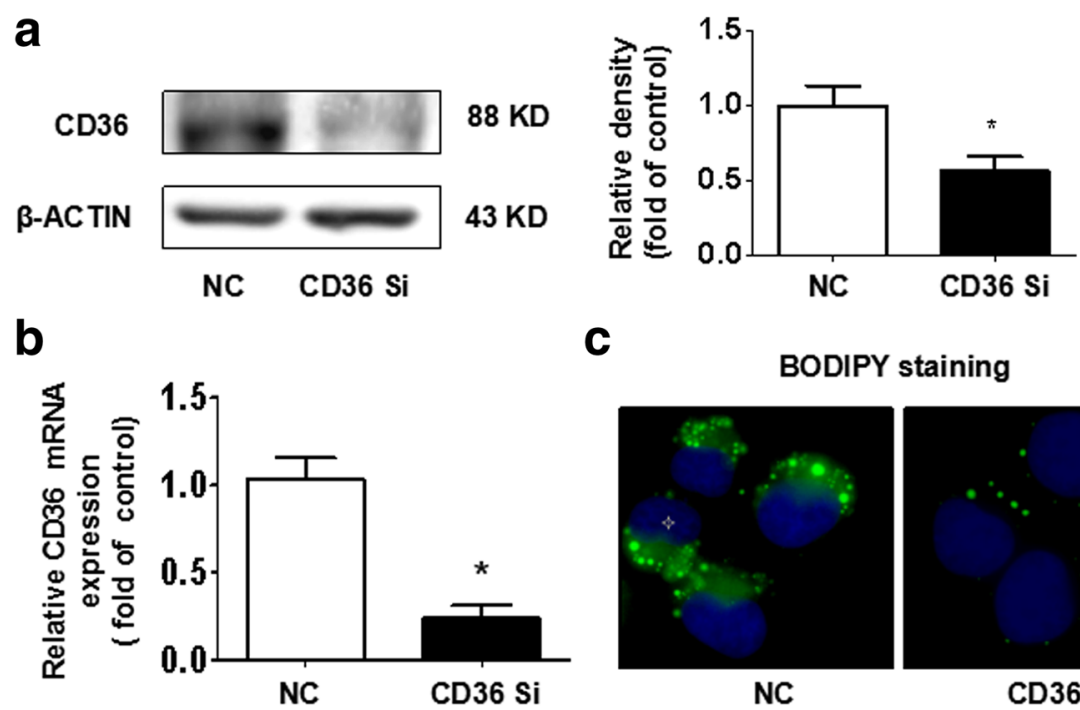

C

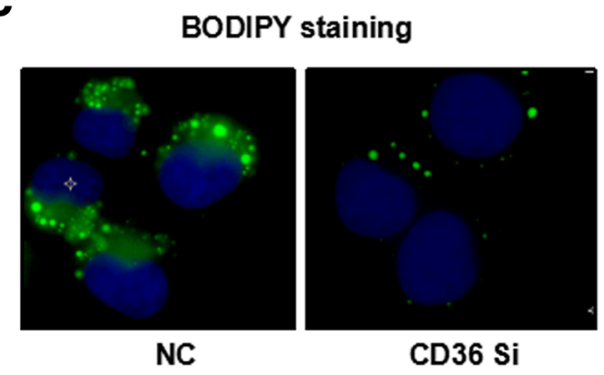

d
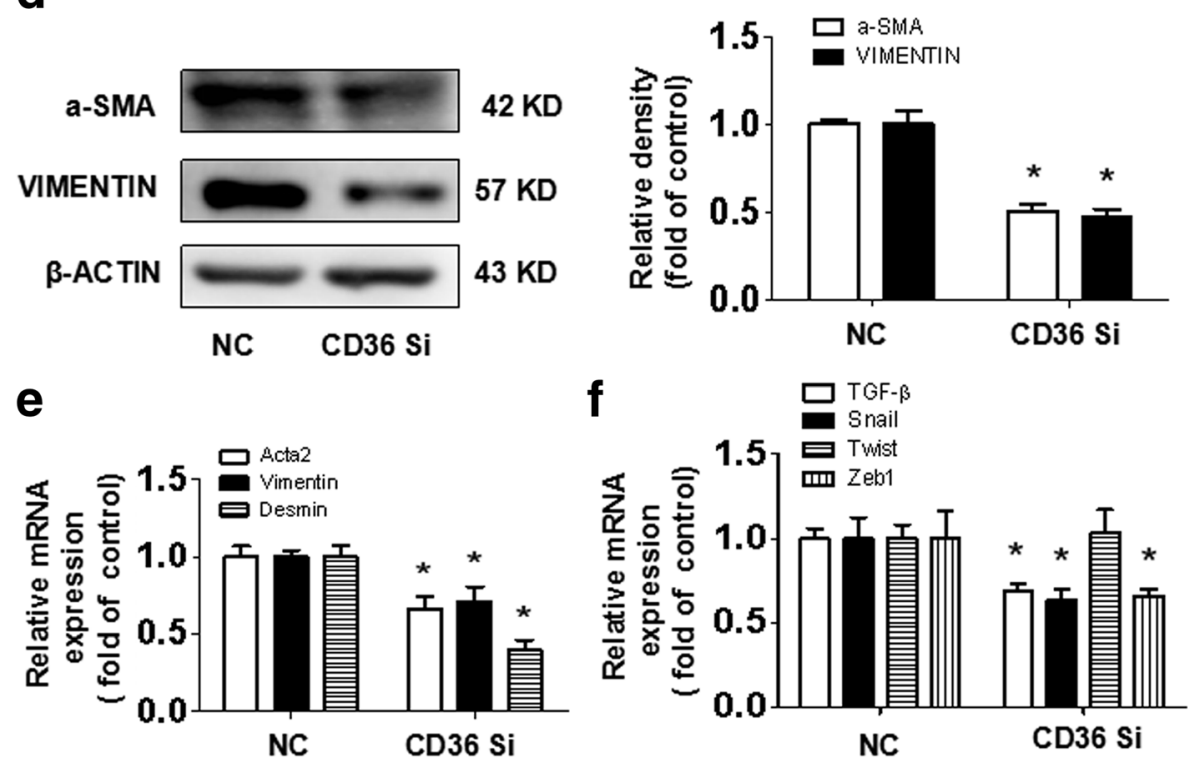

Fig. 3 Effects of the suppression of CD36 on PA-induced hepatocyte activation. CD36-knockdown hepatocytes were treated with $0.2 \mathrm{mmol} / \mathrm{L}$ PA for $48 \mathrm{~h}$. The protein and mRNA expression of CD36 were examined by western blotting (a) and real-time PCR (b). Lipid accumulation was observed by BODIPY staining (original magnification $\times 800$, c). The protein expression of a-SMA and VIMENTIN were examined by western blotting (d). The mRNA expression of Acta2, Vimentin, Desmin and TGF- $\beta$ signaling pathway related genes TGF- $\beta$, Snail, Twist and Zeb1 were determined by real-time PCR $(\mathbf{e}, \mathbf{f})$. The results are depicted as the mean \pm SEM, ${ }^{*} \mathrm{P}<0.05$ versus the NCD group

mediator in the induction of hepatocyte activation, which plays a significant role in the perpetuation of hepatic fibrosis [24]. Our data demonstrated PA increased the mRNA expression of TGF- $\beta$ signaling pathway related gene expression, including TGF- $\beta$, Snail, and Zeb1, and these genes were down-regulated in the CD36 knockdown hepatocytes. Our results suggest that PA may be involved in the regulation of hepatocyte activation through TGF- $\beta$ related pathways, which may be mediated by CD36.
Previous studies have revealed that oxidative stress is closely related to TGF- $\beta$ signaling pathway in liver fibrogenesis [25]. It has been reported that the superabundant accumulation of lipids in hepatocytes could exceed the oxidative capacity of metabolism, causing oxidative stress [26], and CD36 may participate in this process. CD36 is an important mediator of the production of ROS and oxidative stress [27], besides regulation of hepatic fatty acid uptake and TG storage, it also plays a pathological role in the development of various diseases 
a

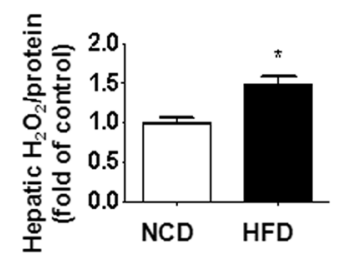

d
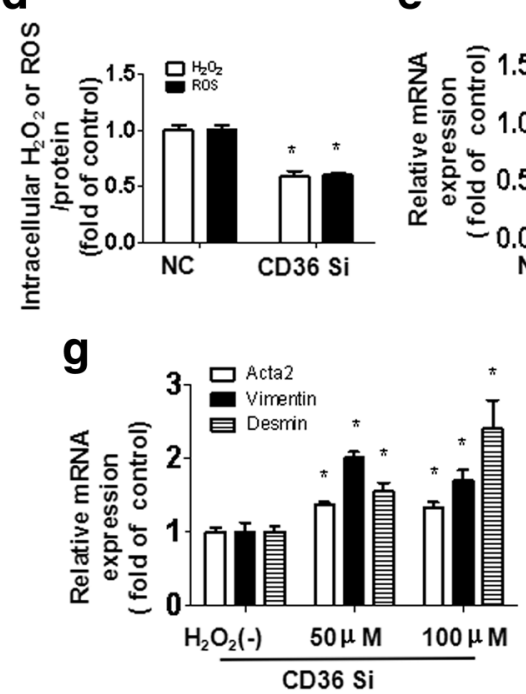

i

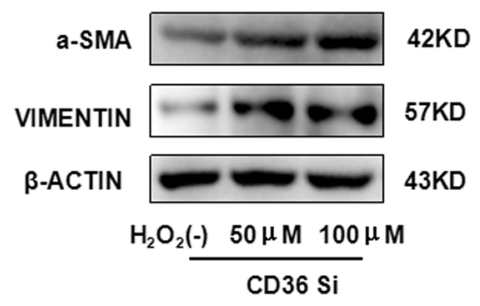

b

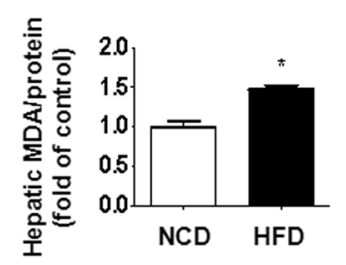

e

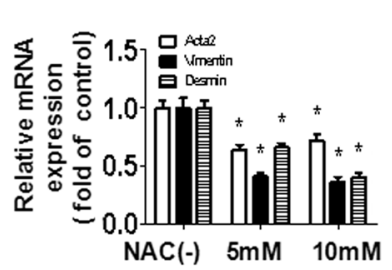

h

$f$
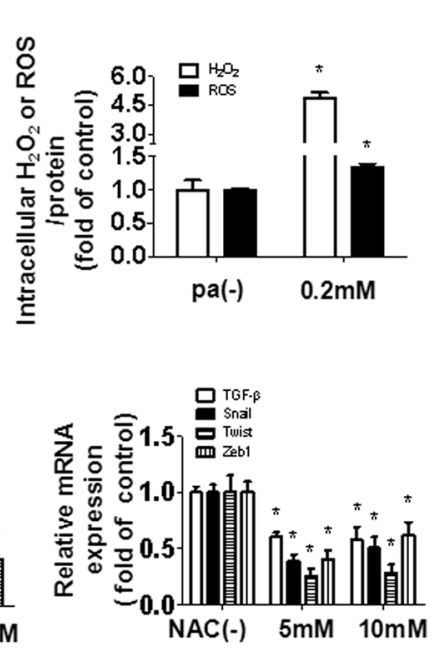
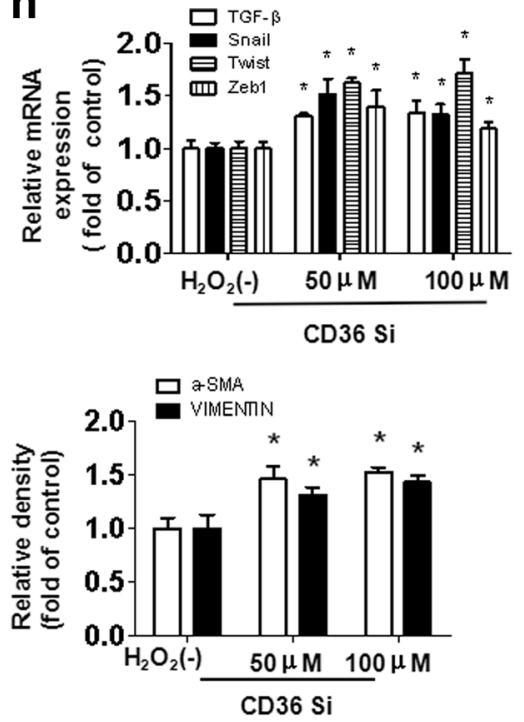

Fig. 4 The oxidative stress involved in PA-induced hepatocyte activation mediated by $C D 36 . \mathrm{H}_{2} \mathrm{O}_{2}$ levels and MDA levels in the livers of mice fed an NCD or HFD for 14 weeks are shown $(\mathbf{a}, \mathbf{b})$. ${ }^{*} \mathrm{P}<0.05$ versus the NCD group. Hepatocytes were incubated in serum-free medium containing $0.2 \mathrm{mmol} / \mathrm{L}$ PA for $48 \mathrm{~h}$, and $\mathrm{H}_{2} \mathrm{O}_{2}$ levels and ROS levels were then determined (c). ${ }^{*} \mathrm{P}<0.05$ versus the PA(-) group. CD36-knockdown hepatocytes were treated with $0.2 \mathrm{mmol} / \mathrm{L} \mathrm{PA}$; after $48 \mathrm{~h}, \mathrm{H}_{2} \mathrm{O}_{2}$ levels and ROS levels were measured (d). ${ }^{*} \mathrm{P}<0.05$ versus the $\mathrm{NC}$ group. Hepatocytes were incubated in serum-free medium containing $0.2 \mathrm{mmol} / \mathrm{L}$ PA for $48 \mathrm{~h}$ alone or with pretreatment of NAC for $2 \mathrm{~h}$. The mRNA expression of Acta2, Vimentin, Desmin and TGF- $\beta$ signaling pathway related genes TGF- $\beta$, Snail, Twist, and Zeb1 were determined by real-time PCR $(\mathbf{e}, \mathbf{f}){ }^{*} \mathrm{P}<0.05$ versus the $\mathrm{NAC}(-)$ group. CD36-knockdown hepatocytes were treated with $0.2 \mathrm{mmol} / \mathrm{L} \mathrm{PA}$ for $48 \mathrm{~h}$ in the absence or presence of $\mathrm{H}_{2} \mathrm{O}_{2}$ for $24 \mathrm{~h}$. The mRNA expression of Acta2, Vimentin, Desmin and TGF- $\beta$ signaling pathway related genes TGF- $\beta$, Snail, Twist, and Zeb1 were determined by real-time PCR $(\mathbf{g}-\mathbf{h})$. The protein expression of a-SMA and VIMENTIN were examined by western blotting (i). *P $<0.05$ versus the $\mathrm{H}_{2} \mathrm{O}_{2}(-)$ group. All results are depicted as the mean $\pm \mathrm{SEM}$

[17]. So, in the present study, we sought to determine whether CD36 is involved in PA-induced hepatocytes activation through oxidative stress. Our data showed that PA or HFD significantly increased the level of oxidative stress in hepatocytes or $\mathrm{C} 57 \mathrm{BL} / 6 \mathrm{~J}$ mice and that these phenotypes were reversed after knockdown of CD36 in hepatocytes. In addition, $\mathrm{N}$-acetylcysteine, an antioxidant, largely inhibited the hepatocyte-activating effects of PA. Furthermore, supplementation of $\mathrm{H}_{2} \mathrm{O}_{2}$ markedly abrogated the improved effects of CD36 knockdown on hepatocyte activation. Taken together, our data suggest that oxidative stress is critical in PA-induced hepatocyte activation and that this activation is mediated by CD36.

Currently, increasing attention has been paid to the involvement of CD36 in liver diseases. In fact, CD36 has been associated with obesity and diabetes in human diseases; Particularly, increased expression of hepatic CD36 is closely related to the development of NAFLD and 
non-alcoholic steatohepatitis (NASH) with insulin resistance [28]. Moreover, our previous work has demonstrated that CD36 plays an important role in balancing hepatic ROS and regulating macrophage infiltration, which could be a new potential therapeutic strategy to prevent NASH development [29]. Hence, it is believed that CD36 is emerging as a novel target for chronic liver disease.

\section{Conclusions}

Our findings demonstrated that PA induces the production of oxidative stress, promoting hepatocyte activation, thus playing an imperative role in the process of hepatic fibrosis. Furthermore, we have uncovered, for the first time, the molecular mechanisms involved in hepatocyte activation via oxidative stress were mediated by CD36, which could be used to acquire novel targets for the prevention and treatment of hepatic fibrosis and may provide a new entry point for researchers studying hepatic fibrosis.

\section{Abbreviations}

BSA: Bovine serum albumin; CD36: Cluster of differentiation; Col: Collagen type; ECM: Extracellular matrix; EMT: Epithelial-mesenchymal transition; FFA: Free fatty acid; $\mathrm{H}_{2} \mathrm{O}_{2}$ : Hydrogen peroxide; HFD: High fat diet: HSCs: Hepatic stellate cells; LCFA: Long-chain fatty acid MDA: Malondialdehyde; NAC: N-acetylcysteine; NAFLD: Non-alcoholic fatty liver disease; NASH: Non-alcoholic steatohepatitis; NCD: Normal chow diet; Ox-LDL: Low-density lipoprotein; PA: Palmitic acid; ROS: Reactive oxygen species; TC: Total cholesterol; TG: Triglyceride; TGF- $\beta$ : Transforming growth factors- $\beta$

\section{Funding}

This research was supported by the National Natural Science Foundation of China (81570517, 31571210 and Key Program, No. 81390354), the Chongqing Research Program of Basic Research and Frontier Technology (No. cstc2015jcyjBX0044), and the Science and Technology Research Program of Chongqing Municipal Education Commission (NO. KJ1702029).

\section{Availability of data and materials}

The authors confirm that all materials described in the manuscript are fully available to any scientist wishing to use them, without restriction.

\section{Authors' contributions \\ $J L, P Y, L Z, L W$ and $Y C$ performed experiments; WT, XYZ and CXS analyzed data and prepared figures; $J L$ and PY drafted manuscript; $J$, PY and YXC performed conception and design of research; $\mathrm{SH}$ and GQZ edited and revised manuscript. YXC and XZR approved final version of manuscript. All authors read and approved the final manuscript.}

\section{Ethics approval and consent to participate}

Mouse care and experimental procedures were approved by the Animal Care Committees at Chongqing Medical University.

\section{Consent for publication}

All authors agree to publish this article in the journal of Lipids in Health and Disease.

\section{Competing interests}

The authors declare that they have no competing interests.

\section{Publisher's Note}

Springer Nature remains neutral with regard to jurisdictional claims in published maps and institutional affiliations.

\section{Author details}

${ }^{1}$ Centre for Lipid Research \& Key Laboratory of Molecular Biology for Infectious Diseases (Ministry of Education), Institute for Viral Hepatitis, Department of Infectious Diseases, The Second Affiliated Hospital,

Chongqing Medical University, Chongqing 400016, China. ${ }^{2}$ Department of Gastroenterology, Chongqing Hospital of Traditional Chinese Medicine, Chongqing 400021, China. ${ }^{3}$ Department of Gastroenterology, The Second Affiliated Hospital, Chongqing Medical University, Chongqing 400010, China. ${ }^{4} J o h n$ Moorhead Research Laboratory, Centre for Nephrology, University College London Medical School, Royal Free Campus, University College London, NW3 2PF, London, UK. ${ }^{5}$ The Collaborative Innovation Center for Diagnosis and Treatment of Infectious Diseases (CCID), Zhejiang University, Hangzhou 310058, China.

Received: 11 April 2018 Accepted: 28 May 2018

Published online: 17 July 2018

\section{References}

1. Zhou WC, Zhang QB, Qiao L. Pathogenesis of liver cirrhosis. World J Gastroenterol. 2014;20(23):7312-24

2. Czaja AJ. Hepatic inflammation and progressive liver fibrosis in chronic liver disease. World J Gastroenterol. 2014;20(10):2515-32.

3. Tennakoon AH, Izawa T, Kuwamura M, Yamate J. Pathogenesis of type 2 epithelial to mesenchymal transition (EMT) in renal and hepatic fibrosis. J Clin Medicine. 2016;5(4):1-16

4. Chen AP. Acetaldehyde stimulates the activation of latent transforming growth factor- $\beta 1$ and induces expression of the type II receptor of the cytokine in rat cultured hepatic stellate cells. Biochem J. 2002;368:683-93.

5. Zhao YL, Zhu RT, Sun YL. Epithelial-mesenchymal transition in liver fibrosis. Biomedical Reports. 2016:4:269-74.

6. Lua I, Asahina K. The role of mesothelial cells in liver development, injury, and regeneration. Gut and Liver. 2016;10(2):166-76.

7. Friedman SL. Stellate cells: a moving target in hepatic Fibrogenesis. Hepatology. 2004;40(5):1041-3.

8. Wu K Ye $\mathrm{CH}_{\text {, Lin L }}$ et al. Inhibiting miR-21 attenuates experimental hepatic fibrosis by suppressing both the ERK1 pathway in HSC and hepatocyte EMT. Clin Science. 2016:130:1469-80.

9. Fausther M, Lavoie EG, Dranoff JA. Contribution of Myofibroblasts of different origins to liver fibrosis. Curr Pathobiol Rep. 2013;1(3):225-30.

10. Kim SM, Choi JE, et al. RAR-related orphan receptor gamma (RORg)mediates epithelial-mesenchymal transition of hepatocytes during hepatic fibrosis. J Cell Biochem. 2017;118:2026-36.

11. Kaimori A, Potter JJ, Choti M, et al. Histone deacetylase inhibition suppresses the transforming growth factor $\beta 1$-induced epithelial-tomesenchymal transition in hepatocytes. Hepatology. 2010;52(3):1033-45.

12. Kawano YK, Cohen DE. Mechanisms of hepatic triglyceride accumulation in non-alcoholic fatty liver disease. J Gastroenterol. 2013;48:434-41.

13. Angulo P, Keach JC, Batts KP. Independent predictors of liver fibrosis in patients with nonalcoholic steatohepatitis. Hepatology. 1999;30(6):1356-62.

14. Tomita K, Teratani T, Suzuki T, Shimizu M. Free cholesterol accumulation in hepatic stellate cells: mechanism of liver fibrosis aggravation in nonalcoholic steatohepatitis in mice. Hepatology. 2014;59:154-69.

15. Bechmann LP, Zahn D, Gieseler RK. Resveratrol amplifies profibrogenic effects of free fatty acids on human hepatic stellate cells. Hepatol Res. 2009; 39(6):601-8.

16. Park YM. CD36, a scavenger receptor implicated in atherosclerosis. Exp Mol Medicine. 2014;46:e99.

17. Nergiz-Unal R, Rademakers T, et al. CD36 as a multiple-ligand signaling receptor in Atherothrombosis. Car Hem Med Chemistry. 2011:9(1):1-9.

18. Huang J, Zhao L, Yang P, et al. Genome-wide transcriptome analysis of CD36 overexpression in HepG2.2.15 cells to explore its regulatory role in metabolism and the hepatitis B virus life cycle. PLoS One. 2016;11(10): e0164787.

19. Scihneiderhan W, Schmid-kotsas A, et al. Oxidized low-density lipoproteins bind to the scavenger receptor, CD36, of hepatic stellate cells and stimulate extracellular matrix synthesis. Hepatology. 2001;34(4):729-37.

20. Scicchitanoa P, Camelib M, Maiello M, et al. Nutraceuticals and dyslipidaemia: beyond the common therapeutics. J Funct Foods. 2014;6:11-32.

21. Zhao L, Zhong S, Qu HY, et al. Chronic inflammation aggravates metabolic disorders of hepatic fatty acids in high-fat diet-induced obese mice. Sci Rep. 2014;5:10222 
22. Nitta T, Kim JS, Mohuczy D, et al. Murine cirrhosis induces hepatocyte epithelial mesenchymal transition and alterations in survival signaling pathways. Hepatology. 2008;48(3):909-19.

23. Kalluri $R$, Weinberg RA. The basics of epithelial-mesenchymal transition. J Clin Invest. 2009;119:1420-8.

24. Kaimori A, Potter J, Kaimori JY, et al. Transforming growth factor- $\beta 1$ induces an epithelial-to-mesenchymal transition state in mouse hepatocytes in vitro. J Biol Chem. 2007;282(30):22089-101.

25. Tsukamoto $\mathrm{H}$, Horne $\mathrm{W}$, et al. Experimental liver cirrhosis induced by alcohol and iron. J Clin Invest. 1995;96:620-30.

26. Spahis $S$, Delvin $E$, Borys $J M$, et al. Oxidative stress as a critical factor in nonalcoholic fatty liver disease pathogenesis. Anti Red Signaling. 2016; 10:e6776.

27. Li W, Febbraio M, Reddy SP, et al. CD36 participates in a signaling pathway that regulates ROS formation in murine VSMCS.J Clin Invest. 2010:120(11): 3996-4006.

28. Colina MEM, Campos SS, et al. Hepatic fatty acid translocase CD36 upregulation is associated with insulin resistance, hyperinsulinaemia and increased steatosis in non-alcoholic steatohepatitis and chronic hepatitis C. Hepatology. 2011;60:1394-402.

29. Zhong S, Zhao L, Wang Y, et al. Cluster of differentiation 36 deficiency aggravates macrophage infiltration and hepatic inflammation by upregulating monocyte chemotactic protein-1 expression of hepatocytes through histone deacetylase 2-dependent pathway. Antioxid Redox Signal. 2017;27(4):201-14.

Ready to submit your research? Choose BMC and benefit from:

- fast, convenient online submission

- thorough peer review by experienced researchers in your field

- rapid publication on acceptance

- support for research data, including large and complex data types

- gold Open Access which fosters wider collaboration and increased citations

- maximum visibility for your research: over $100 \mathrm{M}$ website views per year

At BMC, research is always in progress.

Learn more biomedcentral.com/submissions 